\title{
Adolescente varón con distonía abdominal secundaria al uso de haloperidol y confundida con abdomen agudo
}

\section{Male adolescent with abdominal dystonia secondary to the use of haloperidol and misdiagnosed as an acute abdomen syndrome}

\author{
Lizardo Cruzado ${ }^{1,2}, 2$ Patricia Núñez-Moscoso ${ }^{1 \mathrm{a}, 2}$, Lucy Chang-Torres ${ }^{1,36}$, \\ Humberto Maldonado-Ruiz',2b. \\ 1 Instituto Nacional de Salud Mental Honorio Delgado-Hideyo Noguchi. Lima, Perú \\ 2 Facultad de Medicina. Universidad Peruana Cayetano Heredia. Lima, Perú. \\ 3 Facultad de Medicina. Universidad Nacional Mayor de San Marcos. Lima, Perú. \\ a Psiquíatra, b Ex médico residente de Psiquiatría.
}

Correspondencia Lizardo Cruzado

lizardo.cruzado.d@upch.pe

Recibido: 09/10/2017

Arbitrado por pares

Aprobado: 07/02/2018

Citar como: Cruzado L, NúnezMoscoso P, Chang-Torres L, Maldonado-Ruiz H. Adolescente varón con distonía abdominal secundaria al uso de haloperidol y confundida con abdomen agudo. Acta Med Peru. 2018;35(1):71-4

\section{RESUMEN}

En los últimos años se ha incrementado el uso de psicofármacos, específicamente antipsicóticos, para tratar cuadros no psicóticos en niños y adolescentes. El mayor uso de los antipsicóticos atípicos en desmedro de los clásicos ha influido en un menor conocimiento de sus usuales efectos extrapiramidales. Reportamos el caso de un adolescente varón de 15 años con diagnóstico de esquizofrenia paranoide quien presentó distonía abdominal luego de tratarlo con haloperidol. Atendido bajo la presunción de abdomen agudo, fue sometido a una serie de exámenes auxiliares que resultaron normales. Posteriormente presentó distonía cervical y agitación psicomotriz. Luego de cambiar la medicación inicial por ziprasidona y biperideno, el paciente logró la recuperación y fue dado de alta con el cuadro psicótico controlado. Deben reconocerse los efectos secundarios de los antipsicóticos y considerar su uso solo cuando no haya otras alternativas viables. La distonía de músculos abdominales debe incluirse en el diagnóstico diferencial del abdomen agudo.

Palabras clave: Distonía; Antipsicóticos; Abdomen agudo (fuente: DeCS BIREME).

\section{ABSTRACT}

In the past few years, the use of psychotropic drugs, especially anti-psychotics, has increased, for the therapy of non-psychotic conditions in children and adolescents. The more frequent use of atypical anti-psychotic drugs replacing the classical ones has influenced in having less knowledge of their extra-pyramidal effects. We report the case of a 15 -year old male adolescent diagnosed with paranoid schizophrenia who presented with abdominal dystonia after receiving haloperidol. He was taken care of with a presumptive diagnosis of acute abdomen syndrome, and he underwent some tests that turned out to have normal results. Later, he developed cervical dystonia and psychomotor agitation. After the initial medication was replaced with ziprasidone and biperiden, the patient fully recovered and he was discharged with his psychotic condition under control. Side effects of anti-psychotic drugs should always be recognized, and its use is to be considered only when no other viable option is available. Abdominal muscle dystonia should be included in the differential diagnosis of the acute abdominal syndrome Keywords: Dystonia; Antipsychotics agents; Abdomen, acute (source: MeSH NLM). 


\section{INTRODUCCIÓN}

La distonía es un síndrome caracterizado por contracciones sostenidas y repetitivas de músculos agonistas y antagonistas que pueden llevar a posturas anormales y que se acompañan usualmente de dolor intenso y ansiedad ${ }^{[1]}$. Cuando el paciente no tenga antecedente de enfermedades neurodegenerativas preexistentes, la distonía secundaria al uso de fármacos es la más frecuente ${ }^{[2]}$.

La distonía aguda es un fenómeno usualmente asociado al uso de neurolépticos o antipsicóticos clásicos (se reporta una prevalencia de $10-30 \%)^{[2]}$. En la actualidad, este efecto secundario es menos frecuente por el uso mayoritario de antipsicóticos atípicos ${ }^{[3]}$. Dada la menor posibilidad de entrenamiento de los médicos jóvenes con el uso de neurolépticos, es probable que hoy en día sea más factible que se soslayen cuadros de distonía y resulten confundidos con otras patologías ${ }^{[4]}$.

Reportamos el caso de un adolescente varón de 15 años de edad con diagnóstico de esquizofrenia quien acudió a establecimiento de salud por presentar un cuadro de distonía de los músculos abdominales que fue diagnosticada equivocadamente como un síndrome de abdomen agudo. La distonía solo se resolvió a posteriori y de modo incidental. Hasta donde conocemos, no existen casos similares que fueran reportados previamente.

\section{REPORTE DE CASO}

Paciente varón de 15 años de edad con antecedente familiar de esquizofrenia. Tres meses antes del ingreso a emergencia, refirió tener una intensa halitosis que lo motivó a lavarse los dientes varias veces al día y acudir a diversos dentistas, los cuales descartaron su presencia. Disconforme con el diagnóstico, acusó a su madre de confabularse con los dentistas para hacerle daño y no curarlo. Dejó de acudir al colegio pues creía que se burlaban de él.

Dos meses después, afirmó que la comida casera era desagradable y sospechaba que añadían veneno a su plato. Una semana antes de acudir a emergencia, dejó de asearse y presentó conducta desorganizada: se desnudaba en público e intentó salir de su hogar en la madrugada.

Fue llevado maniatado al departamento de emergencia psiquiátrica. Se mostraba en estado hiperalerta, irritable y profería palabras soeces; su lenguaje era fluido y coherente; presentaba delusiones de daño respecto a sus familiares y negó toda la información proporcionada por ellos. Aceptó tener alucinaciones auditivas pero no precisó su contenido aunque carecía de conciencia de enfermedad.

Se le diagnosticó con esquizofrenia paranoide según los criterios de la CIE-10. Se le aplicó haloperidol $5 \mathrm{mg}$ por vía intramuscular (IM) y diazepam $10 \mathrm{mg}$ por vía endovenosa (EV); no obstante, seguía hostil y agresivo aún luego de tres horas de aplicación, por lo que se le aplicó una nueva dosis de haloperidol $5 \mathrm{mg} \mathrm{IM}$ y quedó en observación con indicación de haloperidol $5 \mathrm{mg}$ IM cada 12 horas.

Al día siguiente, antes de desayunar, refirió la presencia de dolor agudo e intermitente en la región umbilical, que no irradiaba, de 45 minutos de evolución. El paciente se ubicó en posición antálgica (flexionado sobre sí mismo y en decúbito lateral izquierdo), estaba pálido, sudoroso y quejumbroso. Su presión arterial fue de $110 / 60 \mathrm{mmHg}$, frecuencia cardiaca de 138 latidos por minuto, temperatura axilar de $37^{\circ} \mathrm{C}$ y una saturación de $\mathrm{O}_{2}$ de $97 \%$. La evaluación física del abdomen fue difícil debido a la intensa sensibilidad y a que el paciente no podía permanecer por mucho tiempo en decúbito dorsal. Se verificaron ruidos hidroaéreos a la auscultación y rigidez a la palpación superficial y profunda.

Por causa del dolor abdominal intenso se trasladó al paciente a un hospital general para descartar un posible abdomen agudo. Luego de aproximadamente 30 minutos, el dolor abdominal disminuyó parcialmente de intensidad. Se efectuaron exámenes de laboratorio (citometría, bioquímica sanguínea, metabolitos de sustancias psicoactivas y examen de orina) y de imágenes (radiografía y ecografía abdominal) resultando todos normales.

Durante la espera de los resultados, el paciente presentó distonía cervical (flexión lateral izquierda) que conllevó a un episodio de agitación psicomotriz, molestias que cedieron al uso de diazepam $20 \mathrm{mg}$ EV a bolo lento y biperideno $5 \mathrm{mg} \mathrm{IM}$. Este suceso llevó a la sospecha de que el dolor abdominal y la distonía cervical compartían la misma etiología; es decir, un efecto secundario al uso de neurolépticos.

Tras su retorno a nuestro hospital, el paciente continuaba con marcada inquietud psicomotriz e irritabilidad. Se suspendió la administración de haloperidol y se indicó ziprasidona 20 mg IM y midazolam $5 \mathrm{mg} \mathrm{IM}$, con rápido efecto sedativo. Siguió recibiendo ziprasidona $20 \mathrm{mg}$ IM cada 12 horas durante los tres días de su estancia en observación más biperideno $4 \mathrm{mg}$ diarios por vía oral y no volvió a presentar molestias similares. Salió de alta con atenuación de su cuadro psicótico y continuó su tratamiento por consultorio externo.

\section{DISCUSIÓN}

La distonía aguda se ha reportado con el uso de todos los antipsicóticos y otros fármacos antagonistas de dopamina (metoclopramida, flunarizina, cinarizina y domperidona), aunque también con algunos antidepresivos ${ }^{[5]}$. La distonía aguda es el más precoz de los efectos extrapiramidales, ya sea luego de iniciar el consumo de antipsicóticos - casi el $100 \%$ de los casos ocurre dentro de los primeros cinco días - o cuando haya un incremento en la dosis; su frecuencia varía, aunque es mayor cuando se utilizan antipsicóticos parenterales y de alta potencia como el haloperidol, cuando puede presentarse hasta en el 30$60 \%$ de los pacientes ${ }^{[1-3]}$. 
Las distonías localizadas en el cuello son las más frecuentes (30\%), seguidas de mandíbula y lengua (17\%). Los principales factores de riesgo para la distonía aguda por antipsicóticos son: ser de sexo masculino, edad adolescente y juvenil (menos de 30 años), antecedente personal y familiar de distonía aguda, consumo de cocaína y algunas patologías sistémicas como la deshidratación y la infección por VIH, entre otras ${ }^{[1,2,5]}$.

Las patologías a considerar dentro del diagnóstico diferencial son los cuadros conversivos, tétanos, hipocalcemia, hipomagnesemia, anafilaxis y hasta crisis de epilepsia del lóbulo temporal ${ }^{[6]}$. Usualmente, el antecedente reciente de uso de un fármaco antidopaminérgico posibilita el apropiado diagnóstico diferencial.

Se han propuesto dos teorías respecto a la base fisiopatológica de la distonía aguda: a) la hipofunción dopaminérgica provocada por el bloqueo farmacológico conlleva a una hiperfunción relativa de la vía colinérgica -ambas se hallarían en equilibrio en las vías subcorticales extrapiramidales-, de aquí devendría la utilidad de los anticolinérgicos en el manejo de la distonía aguda pues restablecería dicho equilibrio; b) una hiperfunción dopaminérgica paradójica debido al bloqueo de los receptores dopaminérgicos presinápticos: al bajar los niveles plasmáticos del fármaco antidopaminérgico, los receptores de dopamina supersensibilizados se expondrían a la liberación natural de dopamina de los terminales presinápticos, esto explicaría que muchas de las distonías se presentan al bajar los niveles circulantes de la medicación antidopaminérgica ${ }^{[1,2]}$.

En época reciente se ha incrementado el empleo de antipsicóticos de segunda generación tanto en niños como adolescentes para tratar enfermedades de naturaleza no estrictamente psicótica como trastorno bipolar, déficit de atención e hiperactividad, autismo, retraso mental y trastornos de conducta ${ }^{[3,6,7]}$. Si bien estos antipsicóticos tienen menos riesgo de provocar una distonía, se ha criticado en los prescriptores una falsa sensación de seguridad al usarlos, creyendo que no hay posibilidad de que se presenten efectos secundarios ${ }^{[3]}$.

Aunque la presentación más frecuente de la distonía aguda consiste en la contractura de grupos musculares del eje axial (cabeza y cuello), existen dos circunstancias en las que pueden presentarse complicaciones adicionales: 1) cuando la distonía afecta la vía aérea superior por contractura muscular a nivel faríngeo o laríngeo, circunstancia en la que nos hallamos ante una emergencia médica que debe resolverse con el uso de biperideno u otro anticolinérgico $\mathrm{EV}^{[8,9]}$, y 2) cuando la distonía de músculos masticatorios y faciales llega a producir luxación de la articulación temporomandibular (uni o bilateral): en este caso puede haber problemas de diagnóstico diferencial y la interconsulta al cirujano maxilofacial es perentoria ${ }^{[10]}$.
Existen pocas investigaciones sistemáticas sobre distonía por antipsicóticos en niños y adolescentes ${ }^{[11]}$; se ha estimado que los efectos extrapiramidales en general podrían ser tanto o más frecuentes en este grupo etario que en adultos ${ }^{[7]}$. Una reciente investigación registró durante cinco años la presentación de distonía aguda y halló que los más afectados fueron adolescentes de entre 13 y 18 años-explicable por un mayor tono dopaminérgico que en niños-y los agentes causantes fueron por igual los antipsicóticos (haloperidol, sobre todo) y antieméticos, ambos a dosis terapéuticas inclusive; el compromiso de músculos cervicales fue el más frecuente y no se registró ningún caso de distonía de músculos abdominales, aunque sí cuatro de distonía faringolaríngea ${ }^{[6] .}$

Las causas más frecuentes de dolor abdominal agudo en adolescentes son la apendicitis aguda, los traumatismos abdominales, la obstrucción intestinal por bridas y, en adolescentes mujeres, las patologías ováricas. La torsión testicular puede provocar dolor abdominal pero siempre con dolor escrotal. La llamada "migraña abdominal" es esencialmente un cuadro recurrente y de inicio en la infancia ${ }^{[12]}$. Debe señalarse que estos diagnósticos diferenciales no mostraban las características del dolor abdominal de nuestro paciente: su intensidad y evolución hiperaguda, además de la dificultad para el examen físico y la anamnesis -por hallarse psicótico-motivaron el pronto traslado a una emergencia médica.

Los efectos secundarios de tipo motor se hallan entre los más frecuentes efectos iatrogénicos de índole neurológica ${ }^{[5]}$. En la literatura psiquiátrica, los casos de distonía de músculos abdominales son escasos ${ }^{[13]}$. No sabemos de ningún caso reportado de distonía abdominal que genere confusión con un síndrome de abdomen agudo. A partir de nuestro reporte, sugerimos que en niños y adolescentes el uso de medicamentos bloqueadores de dopamina sea restringido a casos en que no exista ningún medicamento de mejor perfil de efectos secundarios, dado que los efectos colaterales de tipo motor son bastante frecuentes con el uso de antipsicóticos y otros fármacos bloqueadores de dopamina.

Se ha señalado que el desarrollo de distonía aguda afecta la relación médico- paciente por lo que debe evitarse su aparición en la medida de lo posible ${ }^{[14]}$. Adicionalmente, debe recordarse que los efectos secundarios farmacológicos requieren un alto índice de sospecha para su detección ${ }^{[15]}$.

Una vez detectada una distonía aguda, usualmente el manejo con un anticolinérgico brinda mejoría rápida aunque, dada la vida media larga de varios fármacos antidopaminérgicos, debiera prolongarse por al menos algunos días la administración del anticolinérgico pues una sola dosis del medicamento bloqueador de dopamina podría producir más de un episodio de distonía ${ }^{[1,5]}$. 


\section{REFERENCIAS BIBLIOGRÁFICAS}

1. Mehta SH, Morgan JC, Sethi KD. Drug-induced movement disorders. Neurol Clin. 2015;33(1):153-74.

2. Annamalai A. Medical management of psychotropic side effects. Cham: Springer International Publishing; 2017.

3. García-Amador M, Merchán-Naranjo J, Tapia C, Moreno C, Castro-Fornieles J, Baeza I, et al. Neurological adverse effects of antipsychotics in children and adolescents. J Clin Psychopharmacol. 2015;35(6):686-93.

4. Dibben CRM, Khandaker GM, Underwood BR, O'Loughlin C, Keep C, Mann L, et al. First generation antipsychotics: not gone but forgotten. BJPsych Bulletin. 2016;40(2):93-6.

5. Rodnitzky RL. Drug-induced movement disorders in children and adolescents. Expert Opin Drug Saf. 2005;4(1):91-102.

6. Derinoz O, Caglar AA. Drug-induced movement disorders in children at paediatric emergency department: 'dystonia'. Emerg Med J. 2013;30(2):130-3.

7. Carbon M, Kapoor S, Sheridan E, Al-Jadiri A, Azzo S, Sarkaria T, et al. Neuromotor adverse effects in 342 youth during 12 weeks of naturalistic treatment with 5 second-generation antipsychotics. J Am Acad Child Adolesc Psychiatry. 2015;54(9):718-27.
8. Kanburoglu MK, Derinoz O, Cizmeci MN, Havali C. Is acute dystonia an emergency? Sometimes, it really is! Pediatr Emer Care. 2013;29(3):380-2.

9. Cruzado L, Rivera-Encinas MT, Revilla-Zúñiga JA, SánchezFernández M. Obstrucción respiratoria alta secundaria a distonía laríngea recurrente inducida por haloperidol y ziprasidona. Rev Neuropsiquiatr. 2015;78(3):176-81.

10. Cruzado L, Núñez-Moscoso P, Garibay-Huamaní M, Villar-Salas A. Luxación témporo-mandibular recurrente y secundaria a distonía por antipsicóticos. Rev Neuropsiquiatr. 2016;79(3):180-5.

11. Gilbert DL. Drug-induced movement disorders in children. Ann NY Acad Sci. 2008;1142:72-84.

12. Errázuriz G, Corona F. Dolor abdominal de origen orgánico en niños y adolescentes. Rev Med Clin Condes. 2011;22(2):168-75.

13. Wichowicz HM, Jakuszkowiak-Wojten K, Sławek J, Sołtan W, Cubała WJ, Landowski J. [Abdominal dystonia in a patient with schizophrenia: a case report]. Psychiatr Pol. 2009;43(6):751-60. [Article in Polish]

14. Van Harten PN, Hoek HW, Kahn RS. Acute dystonia induced by drug treatment. BMJ. 1999;319(7210):623-6.

15. Park HW, Kwak JR, Lee JS. Clinical characteristics of acute druginduced dystonia in pediatric patients. Clin Exp Emerg Med. 2017:4(3):133-137.

\title{
Las ediciones anteriores de Acta Médica Peruana están disponibles en:
}

\author{
www.redalyc.org
}

